\title{
Un moment crucial dans la pratique médicale
}

\section{Jean Martin}

Dr med., membre de la rédaction

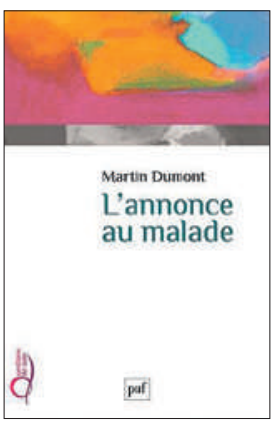

"L'annonce d'une maladie est une situation intense: pour le patient bouleversé par la nouvelle, pour le médecin et pour l'entourage qui l'apprendra. Elle est scandaleuse car les mots, qui s'opposent habituellement à la violence, ici la portent».

Ce petit ouvrage traite du moment majeur de la relation médecin-malade qu'est la communication d'un diagnostic grave voire funeste. Son auteur est un agrégé de philosophie, qui y développe une communication faite devant des spécialistes de la sclérose en plaques - en démontrant bien connaître les fondamentaux de l'éthique médicale. Analyse d'un spécialiste de la communication et du langage susceptible d'intéresser vivement les médecins et d'autres professionnels.

L'ambition du livre est de mieux procéder à ces annonces «non pas en simplifiant par magie mais en refusant d'en rester à l'idée que 'de toute façon, il n'y a pas de bonne façon de faire'.» Le chapitre I décrit les tensions et violences autour de ce moment. Le chapitre II traite des écueils y relatifs, discutant trois éventualités: annonce brutale (une maltraitance), escamotée, ratée. En rappelant Aristote disant que «le véritable courage ne doit céder ni à la témérité, qui mène à des risques déraisonnables, ni à l'excès de prudence qui fait sombrer dans la lâcheté».

Du point de vue du patient. Se souvenir qu'une dimension du traumatisme de l'annonce réside dans le sentiment d'injustice qui s'éveille chez le patient, «auquel il faut s'efforcer d'apporter une réponse, ne serait-ce qu'en reconnaissant qu'il y a bien dans la maladie une forme d'injustice». D'où l'importance de l'attention, de l'empathie. Avec cette phrase de Simone Weil: «Les malheureux n'ont pas besoin d'autre chose en ce monde que d'êtres humains capables de faire attention à eux. Cette capacité est chose très rare, très difficile; c'est presque un miracle.» Formulé succinctement, savoir poser la question: «Quelle est ta douleur?»

Autre aspect: «Les patients savent bien que le fait d'annoncer est difficile pour les médecins. Ils sentent la capacité ou non du médecin à être touché par ce qu'il a à dire et par la personne à qui il le dit [...] Le médecin doit sans cesse être reconduit au caractère inconfortable de la situation, n'étant ni lui-même détruit par l'annonce, ni devenu insensible à celle qu'il doit faire.»

Point spécifique, la difficulté de l'information par le malade à ses proches. "En effet, les patients peuvent subir une stigmatisation du fait de leur maladie, ce qui les rend prudents, alors même que l'annonce à l'entourage permettrait de trouver du soutien, ou d'expliquer des symptômes socialement embarrassants.» On a là une problématique de type "coming out» - qui ne se pose donc pas seulement dans des situations VIH/sida. Le mensonge est une faute médicale. Au chapitre III, il faut vivement recommander la section «Peut-on mentir?». Remarquable démonstration du caractère inacceptable, injustifiable, du mensonge dans la relation de soin - sous réserve de rares situations où il peut être admissible d'introduire un délai (limité), ou d'informer par étapes. Mais la faculté accordée au médecin, dans des déontologies anciennes, de choisir arbitrairement ce qu'il dit et quand, en occultant tout ou partie de la réalité, est inconciliable avec les droits et les intérêts du patient - et le respect qu'on lui doit. "Mentir consiste inéluctablement à amputer autrui d'une part de sa liberté.» Ceci sans compter le fait d'expérience, dans tant de circonstances de vie, que ceux à qui on ment s'en rendent compte, sans oser le dire, et sont alors laissés à leurs incertitudes et leur désarroi.

Après l'annonce. S'efforcer que le patient retrouve, malgré l'annonce funeste, un rapport apaisé au temps qu'il vit: «vie retrouvée, malgré les difficultés, par des biens vécus au présent et non seulement dans les promesses d'une amélioration future peut-être illusoire». Il y a la parole et il y a aussi, qui se poursuit, l'action (le soin), et aussi le silence - souvent nécessaire, bénéfique: «suite aux paroles difficiles de l'annonce, aux paroles consolatrices qui ont pu être prononcées, vient le temps plus silencieux et plus objectif du soin». 